\title{
Implementación de las herramientas tecnológicas como estrategia pedagógica ${ }^{1}$ Implementation of technological
tools as a pedagogical strategy
}

DOI: http://dx.doi.org/10.17981/cultedusoc.9.2.2018.15

Fecha de recepción: 26/06/2018. Fecha de aceptación: 20/09/2018

\author{
Milciades Camargo-Romero ${ }^{2}$ \\ Eliana Ruiz-Martínez; Ramón Abello-Urueta; \\ Sandra Hernandez-Sarmiento; Nielsen Arévalo-Reslen y Olga Ospino-Bustamante \\ C.E.B. Rural de Niñas Isla del Rosario, Pueblo Viejo (Colombia) \\ milcamaro@hotmail.com
}

Para citar este artículo

Camargo-Romero, M., Ruiz-Martínez, E., Abello-Urueta, R., Hernandez-Sarmiento, S., Arévalo-Reslen, N. y Ospino-Bustamante, O. (2018). Implementación de las herramientas tecnológicas como estrategia pedagógica. Cultura. Educación y Sociedad 9(2), 159-167. DOI: http://dx.doi.org/10.17981/cultedusoc.9.2.2018.15

\section{Resumen}

La presente investigación tuvo como objetivo implementar herramientas tecnológicas para mejorar el proceso pedagógico en el C.E.D. Rural de Niñas Isla del Rosario. El desarrollo del estudio se enmarco en autores como Moreira, Rodríguez, bajo una mirada Cuantitativa, desde el paradigma empírico analítico y con un alcance descriptivo. La población participante del estudio fueron los estudiantes y docentes de la IED Mario E Pereira. Para la recolección de la información se empleó una encuesta con preguntas cerradas. Como conclusiones se destaca que Lo resultados permiten mostrar que los estudiantes si conocen de las tecnologías y que ya hacen arte de la vida de los estudiantes y docentes, que les motiva realizar actividades utilizándolas y que manipulas con mayor favorabilidad algunas de estas herramientas, por lo tanto, la escuela debe fundamentar su práctica en la implementación de portales digitales a los cuales la población educativa tenga acceso.

Palabras clave: Investigación, herramientas tecnológicas, procesos pedagógicos, comunidad educativa, practicas pedagógica

\begin{abstract}
The objective of this research was to implement technological tools to improve the pedagogical process in the C.E.D. Rural Girls Rosario Island. The development of the study is framed in authors like Moreira, Rodríguez, under a quantitative view, from the analytical empirical paradigm and with a descriptive scope. The students participating in the study were the students and teachers of the IED Mario E Pereira. For the collection of information, a survey with closed questions was used. As conclusions, it is highlighted that the results allow to show that the students know about the technologies and that they already make art of the students 'and teachers' lives, that motivates them to carry out activities using them and that they manipulate with greater favoribility some of these tools, so Therefore, the school must base its practice on the implementation of digital portals to which the educational population has access.
\end{abstract}

Keywords: Research, technological tools, pedagogical processes, educational community, pedagogical practices

\footnotetext{
${ }^{1}$ Este artículo ha sido derivado del Programa de Fortalecimiento de la Cultura Ciudadana y Democrática CT+I a través de la IEP apoyada en TIC en el Departamento de Magdalena: CICLÓN. Desarrollado con docentes miembros del Grupo de investigación "Los Energéticos" pertenecientes a la Centro de Educación Básica Rural de Niñas, Isla del Rosario Pueblo Viejo.

${ }^{2}$ Líder del grupo de investigación "Los Energéticos".
}

The author; licensee Universidad de la Costa - CUC. 


\section{Introducción}

La educación en Colombia según Palencia, Carvajal \& Rodríguez, (2015) a pesar de transformar significativamente su sistema de educación durante las dos últimas décadas, enfrenta dos desafíos críticos, altos niveles de desigualdad en términos socio económicos, desde los primeros años de educación y un bajo nivel de calidad en los estudiantes y los docentes en el sistema educativo. Es importante saber que el Gobierno ha implementado una variedad de iniciativas (mayor oferta a nivel preescolar, jornada única) para enfrentar las disparidades en cuanto a aprendizaje y acceso a la educación.

Las políticas que viene utilizando han buscado incrementar la participación y mejorar el desempeño escolar de los estudiantes vulnerables y en condiciones de pobreza. La educación es gratuita, modelos educativos flexibles, la educación étnica, la alimentación y el transporte escolar ha contribuido a llegar a los grupos menos favorecidos.

Según el Ministerio de Educación Nacional (MEN)(2016) Colombia ha puesto la Educación como prioridad para mejorar la prosperidad económica social del país, se ha logrado la inversión de más recursos económicos, ha dado grandes pasos para ofrecer una educación incluyente y de calidad, ha alargado el periodo de tiempo que los niños van a la escuela, ha garantizado el ingreso desde edades más tempranas y continúen en la educación superior, haciendo énfasis en los más desfavorecidos, se ha introducido mecanismos de aseguramiento de la calidad, y se está trabajando en el mejoramiento de la profesión docente.

El Centro Educativo Departamental Rural de Niñas Isla del Rosario, Municipio de Pueblo viejo Departamento del
Magdalena, limita por el norte con el mar caribe, por el sur, este y oeste con la Ciénaga Grande de Santa Marta. Esa una región pesquera, la cual comercializa con los diferentes mercados tanto a nivel departamental como a nivel nacional. La pesca es artesanal ya que los métodos utilizados son elaborados por la misma comunidad, tales como atarrayas, chinchorros, pelambres, anzuelos, el garceado $\mathrm{y}$ otros.

La escuela se encuentra situada en el marco de la playa, teniendo a su alrededor la iglesia, la casa de la cultura y la inspección de la policía. La carretera panamericana divide el departamento en dos partes, una que es la zona turística por encontrarse a la orilla de la playa y la zona residencial. Es importante saber que la comunidad, su nivel socio-económico es bajo y hay un alto grado de analfabetismo digital por falta de acceso a internet en sus viviendas, por ende, en el colegio los alumnos tienen oportunidad a acceder a el recurso de internet hoy en día.

En los años anteriores la institución no tenía acceso a internet y había una gran brecha digital a la fecha el gobierno viene trabajando a cerrar esa brecha con programas como kiosco digital para que los alumnos tengan acceso a internet de una forma cómoda a su nivel económico y viendo que para ayudar a mejorar nuestro proceso de enseñanza y aprendizaje nace una necesidad que los alumnos se apoyen con los correos electrónicos y blogs para mejorar nuestra capacidad de comprender, interpretar y elaborar contenidos comunicativos que conlleve al estudiante a ser autónomo, investigativo, democrático y participativo.

Con este proyecto se espera que haya un impacto en la comunidad del C.E.D. Rural de Niñas Isla Del Rosario Pueblo Viejo (Magdalena) para que incorpore 
herramientas tecnológicas que faciliten los procesos pedagógicos y estén a tono con los cambios de la nueva era. Es decir el uso herramientas, tales como: correo electrónico, redes sociales (Facebook), plataformas virtuales y el blog, de alguna manera llevaran a los estudiantes a conectarse con las nuevas tecnologías, momentos históricos que debe agenciar la escuela de hoy. Es decir debe darse a la tarea de innovar sus prácticas con la aplicación de las tecnologías de la información.

Esto consentirá que la institución educativa, se de a la tarea de abrir otros espacios de reflexión, para apuntarle a la tecnología de la información, y este a la vanguardia de lo que plantea el nuevo milenio, una educación centrada en la calidad educativa, con estudiantes aplicando la interactividad de la experimentación.

\section{Uso Educativos de los Blogs}

Los Blogs se caracterizan, por ser otra herramienta, que el maestro de hoy puede utilizarlos en compañía de sus estudiantes. En la relación maestro a alumno los acerca, porque los invita al maestro y al estudiante a escribir, de tal manera que se convierten en escritores de sus propias ideas y pensamiento. El maestro en examinador de sus estudiantes, pues le ofrece la posibilidad de escribir y este revisar, intercambiar ideas, trabajar en equipos, el estudiante para mostrar sus fortalezas y debilidades y seguir avanzando en la parte escritural.

Con la creación de Blogs en las instituciones educativas, sirve de apoyo a la actividad pedagógica, porque se convierte en una herramienta útil de producción intelectual, los estudiantes reafirman sus competencias escriturales y los docentes profundizan en la misma. De tal manera que le apuntan a logro de desarrollo de las competencias lectora-escritora, pues al tiempo debe desarrollar las dos para poder escribir. Esto lleva también a que el docente le exija a los estudiantes de alguna manera deban ampliar sus horizontes para que tengan capacidad de análisis y síntesis. La estrategia de aplicación del Blogs, en la enseñanza aprendizaje, facilita a los estudiantes la mejora de los conocimientos ofreciéndole la posibilidad de realizar proyectos y actividades de aula, optimizando el tiempo, la mejorar de los contenidos académicos, enriqueciéndolos con los medios multimediales como: Videos, sonidos, Imágenes, animaciones, ovas y la Web 2.0. (Blanco, 2005).

La manera como se nutren los Blog, depende de cada de cada uno de los sujetos, quien lo despliega, en el campo de la educación depende la información que desarrolla el maestro y el estudiante. Es decir el contenido que lleva el Blogs, depende del examen que se quiere introducir y la información que se desea orientar. Porque la técnica o plantilla del Blogs como tal, ya está dada, hay que concentre en la información, que se desea contener en la página. Este recurso invita a maestros y estudiantes sin importar el área académica, puedan crear recursos $\mathrm{y}$ contenidos de temas educativos, sin necesidad de instalar aplicaciones o de tener conocimientos de Programación. Edublogs. (Blanco, 2005).

Una de las carteristas importantes de la utilización de estos recursos, como son los llamados Blogs, es que invita a la educación de hoy, a transformar sus prácticas pedagógicas, innovando con nuevas herramientas tecnológicas, dándole la posibilidad de mejorar sus procesos desde la interacción comunicativa con sus estudiantes. Lleva al maestro la innovación a recorrer el camino de la 
innovación como un viaje conexo. (Sáez Vacas, 2005). La importancia del artículo radica, en señalar que esta herramienta es uno de los medios que facilita la competencia lectora escritora, pero no es el definitivo en materia tecnológica. Por qué este proceso tecnológico siempre avanza a la velocidad de la luz y el ser humano debe estar preparado para estos retos que depara la modernidad.

Lo que se pretende con el uso de esta herramienta, es que maestros y docentes hagan uso de ella, de tal manera que la educación de hoy provoque nuevas formas de aprender y una de esta es la aplicación de esta estrategia posibilitadora de cambio.

Existen cifras de datos que denotan la utilización y creación de Blogs, como los llamados directorios de Schoolblogs. com -con unos 4.000 blogs-y los de Bitacoras.com, que actualmente registra más de 300 edublogs en España. Están son experiencias de instituciones educativas de primeras experiencias en este país que han permitido a los departamentos de Comunicación y Periodismo de centros de enseñanza superior, como la Universidad de Navarra (Orihuela, 2004). La Universidad de Málaga (Blanco, 2005). Y la Universidad Carlos III de Madrid (Lara, 2004). Donde se dieron la posibilidad de trabajar con Blogs en determinadas asignaturas, trabajan con sus alumnos y los motivan a crear sus propios Blogs. Este paso facilito de alguna manera el desarrollo integrado de sus programas, para que los estudiantes facilitaran sus aprendizajes. Esta experiencia es un modelo a seguir porque muestra el trabajo interactivo y colaborativo entre maestros y estudiantes, como una manera de aprovechar los recursos y facilitar la enseñanza.

\section{Investigación como estrategia}

pedagógica (IEP)

La I.E.P. surge del replanteamiento que se produce en el siglo XX en el ámbito de la investigación. En primer término, a nivel Epistemológico, porque los postulados del paradigma positivista que hasta esa época dominaban la esfera del ambiente científico son cuestionados de manera firme, por considerarse insuficientes para dar respuesta a las problemáticas sociales que impactan en los grupos humanos, ya que, según esta corriente, las explicaciones de los fenómenos sociales son dadas a través de la postura objetiva del investigador, excluyendo los estados subjetivos del mismo. Para esto, solo se emplean métodos cuantitativos en los procesos investigativos. ( Cochran Lythe y Martin 2006)

La exigencia social de mitad del siglo $\mathrm{XX}$ producto de las grandes movilizaciones y desplazamientos de la posguerra genera un nuevo giro en el campo científico, y es como se llega a la convicción de que las ciencias sociales debían acercarse más al tejido social, hecho que permite la irrupción del paradigma socio - crítico, que recoge información de los dos anteriores, permitiendo la implementación de métodos cuantitativos y cualitativos.

Es justamente en la relación enseñan$\mathrm{za}$ - aprendizaje que se aprecia el alcance e importancia de la I. E. P., concepto que es elaborado a partir de las construcciones de las pedagogías críticas desarrolladas en el contexto Latinoamericano, en especial la pedagogía fundada en la indagación, y en comunión con los enfoques dinamizados desde otras latitudes como el aprendizaje situado, que consiste en que los problemas planteados surgen de la inquietud de los niños o niñas motivados por lo que observan en su entorno. 
Además, existen otras condiciones para su denominación, como la movilización de actores participantes que tienen como punto en común el interés por solucionar la problemática planteada porque los afecta de manera directa y están resueltos a transformar la realidad. Se requiere también, que la educación pública apunte a niveles de excelencia para garantizar a los estudiantes los recursos necesarios para esa transformación de su entorno y la disminución de la brecha de la desigualdad. Igualmente, es primordial la participación activa de docentes que desde su autonomía acepten que hay que transformar la pedagogía y su propia práctica pedagógica (Martínez, 2014).

Según Guerrero, (2016) Las comunidades establecidas son las encargadas de dinamizar el proceso y dar sostenibilidad al ciclo, momento en el que se puede evidenciar el aprendizaje problematizador, en el cual, se parte de la pregunta genuina del niño(a), que da paso a la configuración del problema, porque la pregunta ingenua es reflejo de la manera como el infante entiende lo que le rodea y que a su vez desea transformar, es la expresión del sentido común que problematiza el mundo, para pasar luego a adquirir una conciencia crítica frente a la vida.

Según el autor Duque (2016), la investigación como estrategia pedagógica se da mediante la relación entre la práctica pedagógica y la investigación, brindándoles a las niñas y niños la posibilidad de apropiarse del conocimiento, utilizando herramientas propias de la ciencia, con la mirada puesta en los problemas que brinda el contexto, para dar así respuestas a necesidades sentidas, producto de la situaciones encontradas y de esta manera operacionalizarlas, Ausubel (2001) de esta manera se contribuye a resolver situaciones, desde el punto de vista del objeto de estudio y se apoya con la ciencia, porque se pone en marcha los conocimientos desarrollados en los procesos investigativos, el cual busca innovar, contribuir, revisar enriquecer el discernimiento.

En el ámbito de la educación, por lo menos la escuela debe darse a la tarea, de abrirse a la actividad científica, desde el punto de vista del pensamiento, interacción, la innovación, la justificación dando respuesta al porque, a la aplicación de la enseñanza. Estos elementos examinados a la luz de la experiencia, le aporta al conocimiento del estudiante. Es decir el estudiante entra al mundo de entender los fines de la escuela, desde una visión de cambio, para esto el maestro es la columna de sus estudiantes, los lleva a investigar y una forma de hacerlo es plantearse proyecto de aula, que le den más vida y dinamismo a la práctica docente y al proceso de aprendizaje. En este sentido es el maestro mediador y responsable de llevar a los estudiantes en el mundo de la experimentación, buscando que estos reflexionen en cuanto a la comprensión del mundo y la forma como cambiar esa visión de mundo. Por tanto este maestro relaciona la concepción que tiene de la pedagogía con el campo del saber, por tanto este maestro de hoy necesita replantear sus esquemas como. Schon (1991), para intensificar su rol de maestro, con actividades propias que exige pertenecer a este nuevo milenio.

Cochran, Lythe y Martin (2006) expresan que la existencia de conocimientos y reflexiones se integra con las actuaciones explícitas e implícitas están contenidas en la dimensión cognitivo, así como lo emocional, la teoría y la práctica. Reconociendo que quien enseña como quien aprende dentro de una comunidad, son los llamados a generar el conocimiento local. Es decir están llamados a 
manejar la teoría y la práctica, para luego hacer sus propias reflexiones, análisis y concluir desde lo interpretado.

En la escuela es relevante el uso de la investigación o indagación como metodología para construir un espíritu científico en las niñas y niños, en la que se proponen múltiples caminos para dar respuesta a tales procesos, uno de estos caminos son las buenas prácticas docentes. Debe pasar de un extremo a otro, de tal manera que se convierta en una experiencia en la que interactúa el disfrute, el goce por la indagación, la observación y el diálogo, en la que se privilegia el saber, para dar paso a las inquietudes, hallazgos, descubrimientos, que fusionen de manera coherente el paso a paso para fortalecer el desarrollo curricular de aula.

\section{Integración curricular de las TIC}

Integrar las TIC al currículo en las aulas de clases, reconocen que los estudiantes estén a la vanguardia de las nuevas tecnologías y exige que los docentes implementen practicas novedosas, que requieren cambios en la nueva forma de enseñar y aprender. Por lo tanto los cambios que exigen la manipulación de ordenadores como tabletas, telefonía móvil, videojuegos. Es decir estas ayudas favorecerán a las tecnologías, porque encaminan al estudiante y al maestro adentrarse a los paradigmas que hacen cada vez más que la información les llegue a los sujetos de la educación con prontitud. La educación de hoy exige un maestro que este a tono con dinámicas de clase más activas, que abandonen lo tradicional y le dé cabida a las prácticas pedagógicas, para que los estudiantes formen parte activa del asunto. En este sentido se introduce la TIC al currículo, cuando el maestro desde sus prácticas le da paso a esta para que sean más interactivas, es decir cuando comienza a desarrollar clase y privilegia las TIC en doble vía, es decir las utilizan maestro y estudiantes, estudiantes y maestros. (Torrego, 2012, citado en Azorín Abellán, 2015).

Lo anterior deja ver que la transformación del trabajo de aula, permite cambios de los nuevos escenarios, abriéndole al estudiante las posibilidades de aprendizajes más abiertos, interactivos, ricos en motivación, estimuladores, agenciadores de aprendizajes. (García, Basilotta \& López, 2014, citados en Azorín Abellán, 2015).

\section{Las plataformas virtuales}

Es importante destacar que hay numerosas plataformas virtuales, las cuales tienen la característica de ser un software diseñado para ayudar, a padres de familias, estudiantes y maestros. Esta posibilidad de plataformas orienta la manera de cómo crear, diseñar y evaluar programas académicos que fortifican la calidad educativa y los entornos virtuales de aprendizajes. Es decir las plataformas indican la utilidad del servicio que prestan estos Software. (Lara, 2004). Sin embargo toda plataforma educativa virtual, informa y explica cómo se debe cargar las informaciones, que se pretende utilizar desde los trabajos colaborativos, hasta la aplicación de otras herramientas que fundamentaran y optimizaran el trabajo docente.

Toda plataforma con fines educativos tienen estructura modulares que hace posible su adaptación a la realidad de lo programas curriculares, planteados por el ministerio de educación Nacional (MEN). Por lo tanto las instituciones educativas deben responder a las necesidades que tienen y apuntarle a la investigación 
desde las TIC, como único hecho transformador de la realidad educativa. Por tanto estas instituciones deben darle prioridad a los (PEI), Proyecto Educativo Institucional fortalecer sus cuatro áreas de gestión: gestión administrativa, gestión académica, gestión financiera y gestión comunitaria. Para que de esta manera se puedan mejorar los aprendizajes con la utilidad de las plataformas virtuales. (Lara, 2004).

Las plataformas educativas, como asunto novedoso, hacen parte de las instituciones educativas, para que etas las utilicen como medio de mejora en la estructura de bases de datos y como novedad para matricular estudiantes y sistematización de información importante. Llevándolas a que estén en avanzada información oportuna e información de calidad, en cuanto a programas virtualizados, entrega de informes por periodos, sistematización de la planta de personal. Las plataformas mantienen actualizados a los estudiantes de programas y actividades que se desarrollan al interior de estas, como también de las notas académicas digitalizadas y gestión de la comunicación y la información para padres de familia y comunidad en general.

Por esta razón una plataforma virtual, ofrece a sus usuarios espacios de trabajo colaborativo, en la que se comparten e intercambian contenidos, tareas, actividades en general, e incorporan herramientas de comunicación como (chats, correos, foros de debate, videoconferencias, manejo de ensayos escriturales, blogs, etc.)entre otras.

\section{Metodología}

Este estudio se llevó a cabo desde una mirada cuantitativa, desde el paradigma empírico analítico la realidad de los fenómenos que se estudian en su contexto natural. El alcance de la investigación es descriptivo. Pues tal como lo afirma Martínez (2006) es la investigación cuantitativa la que estudia la realidad en su contexto natural, identificando la naturaleza de la realidad.

\section{Población y muestra}

La población participante del estudio fueron los estudiantes y docentes de la IED Mario E Pereira. Para la recolección de la información se empleó una encuesta con preguntas cerradas. Se tomó una muestra de 20 estudiantes y 10 docentes de la institución educativa.

\section{Instrumentos de recolección de la información}

La recolección de los datos se llevó a cabo con la aplicación de una encuesta de preguntas cerradas para la mayor precisión frente a la información requerida. Asi mismo se tuvo en cuenta la observación y registradas a través de un diario de campo.

\section{Procedimiento.}

Fase (1): Identificación de la problemática a estudiar.

Fase (2): Definición de la población y muestra.

Fase (3): Definición de las variables de estudio.

Fase (4): Fundamentación teórica.

Fase (5): Escogencia de las técnicas e instrumentos de recolección de información. Fase (6): Aplicación de los instrumentos. Fase (7): Análisis y procesamiento de los datos.

Fase (8): Generación de resultados.

Fase (9): Socialización y discusión de los hallazgos encontrados. 


\section{Resultados}

A continuación, se presentan los resultados derivados de la encuesta aplicada a los estudiantes y docentes:

Frente a la primera pregunta sobre si ¿Alguna vez ha manejado una herramienta tecnológica? el $96 \%$ de los encuestados respondió que sí y solo el $6 \%$ respondió que no.

Atendiendo a la segunda pregunta sobre ¿Cuál es la herramienta tecnológica que más utiliza? el $60 \%$ de los participantes afirmó que el teléfono celular, el 30\% que el computador y finalmente el $10 \%$ que la tablets

Con base a la última pregunta que hace alusión a si ¿Le gustaría que en la Institución educativa existiera alguna red social educativa? ¿Cual? El 70\% de los encuestados respondió que un Facebook educativo, mientras que un $25 \%$ mencionó que un canal de you to be educativo, y finalmente un $5 \%$ dijo que un blog educativo.

\section{Conclusión}

Poder comprender el escenario educativo y la multiplicidad de factores que intervienen en el resulta complejo, es por ello que la comunidad educativa Mario E Pereira a la luz del presente proyecto busca fortalecer las estrategias pedagógicas para la apropiación del conocimiento por parte de los estudiantes de un manera novedosa, dinámica recreativa y práctica.

Lo resultados permiten mostrar que los estudiantes si conocen de las tecnologías y que ya hacen arte de la vida de los estudiantes y docentes, que les motiva realizar actividades utilizándolas y que manipulas con mayor favorabilidad algunas de estas herramientas. Por todo lo planteado es el colegio el que tiene como propósito poner a disposición de los maestros y estudiantes un portal educativo interactivo para que se emprendan nuevos métodos de enseñanzas que promuevan cada vez la investigación y se descubran nuevas formas de acceder al conocimiento en tiempo real y con recursos actualizados.

\section{Referencias}

Azorin C. (2015). Integración pedagógica de wix en educación primaria. PixelBit. Revista de Medios y Educación, Vol. 163-177. Recuperado de Ministerio Nacional de Educación (MEN). (2016). Revisión de políticas nacionales de educación: La Educación en Colombia. OCDE. Educación in Colombia (3).http://www.redalyc.org/ articulo.oa? $\mathrm{id}=36841180011$.

Duque, I. (2016). La investigación como estrategia pedagógica en el aula. Compartir aula maestra. Documento Recuperado de: https://compartirpalabramaestra.org/columnas/la-investigacion-como-estrategia-pedagogicaen-el-aula.revista de Pedagogía, vol. 32, núm. 90, enero-junio, 2011, pp. 1343. Universidad Central de Venezuela. Recuperado de: http:/www.redalyc. org/articulo.oa?id=65920055002

Guerrero, H. y Cepeda, M.(2016) Uso de estrategias pedagógicas para el fortalecimiento de la convivencia escolar de jóvenes.

Herrera, B. (2014) Las acciones colectivas en Colombia frente a una realidad global: El derecho de consumo. En revista Justicia, No. 25 - pp. 70-81.

Mejía, M \& Manjarrez, M. (2011). La investigación como estrategia pedagógica una apuesta por construir pedagogías críticas en el siglo xxi. Revista de educación y pedagogía Praxis y Saber. 
Martínez, Royert JC. (2014). Modelo de evaluación del Proceso de Atención de Enfermería. [Tesis doctoral], Universidad del Atlántico, Atlántico, Barranquilla. doi:10.13140/RG.2.2.35792.00000

Mejía, M. \& Manjarrez, M. (2013). La investigación como estrategia pedagógica una propuesta desde el sur. Bogotá: Ediciones desde abajo.

Palencia, E. Carvajal, P. \& Rodríguez, F. (2016) La nomocracia sustrato de las corrientes judicialistas en el derecho colombiano. En Revista de la Facultad de Derecho, No. 41, 191-214. http:// dx.doi.org/10.22187/rfd201628
Sabino, C. (1992): "El proceso de la investigación". Editorial Panapo. Caracas, Venezuela. Tesis de investigación recuperado http://tesisdeinvestig. blogspot.com.co/2014/06/tecnicas-einstrumentos-de.html

Villarroel, F. (2012). El constructivismo. y su papel en la innovación educativa. Revista de Educación y Desarrollo, 20. Enero-marzo de 2012. Recuperado de: www.cucs.udg. $\mathrm{mx} /$ revistas/edu_desarrollo/anteriores/20/020_Villarruel.pdf 